\title{
Patchy particles by Glancing Angle Deposition
}

\author{
Amar B. Pawar and Ilona Kretzschmar* \\ Department of Chemical Engineering, The City College of New York, $140^{\text {th }}$ Street \& \\ Convent Avenue, New York, NY 10031, USA. \\ kretzschmar@ccny.cuny.edu
}

Received....

* Corresponding author - Ilona Kretzschmar, kretzschmar@ccny.cuny.edu

Experimental

Monolayer Fabrication: An ordered close-packed two-dimensional array of $2.4 \pm 0.14$ $\mu \mathrm{m}$ sulfate latex polystyrene particles (Interfacial Dynamics Corporation) is generated on a silicon (110) wafer (University wafer) by continuous convective assembly using a circular acrylic cell. The stock solution of the microparticles (8.4 wt. \%) is diluted 100 times with deionized water and used for the assembly. The acrylic ring (OD $=2 \mathrm{~cm}$ and ID = $1 \mathrm{~cm}$ ) is clamped on a precleaned silicon wafer using a glass slide for the support. The 100 times diluted particle solution is added to the bore and the set up is kept inside an oven for drying at $40^{\circ} \mathrm{C}$ until all solvent has evaporated. The amount of particle solution added to the cell is necessary to form the hexagonally close-packed particle monolayer within the bore of the cell. 
Metal Evaporation: Gold vapor deposition on the colloidal crystals is performed inside a bench-top vacuum metal evaporation system (Cressington 308 R, Ted Pella, Inc.) at a pressure of $10^{-6}$ mbar. Patches with a thickness of $\sim 20 \mathrm{~nm}$ are obtained with a deposition rate of $\sim 1 \mathrm{~nm} / \mathrm{s}$ measured by a quartz crystal monitor. A 0.008 inch diameter gold wire (99.99 \% Au) from Ted Pella, Inc. is used for the evaporation. The angle of incidence of Au vapor flow, $\theta$, measured from the substrate ( $90^{\circ}$ being vertical to sample), is adjusted by tilting the sample stage in the vacuum chamber. The effect of the angle of incidence on the patch geometry is studied by varying $\theta$ from $30^{\circ}$ to $10^{\circ}$ and $2^{\circ}$.

Imaging: Following the evaporation, the sample is inserted into a variable-pressure scanning electron microscope (EVO40 Zeiss). The imaging of the Au patterned sulfated latex particles is carried out at $\sim 10^{-6}$ mbar. The reference monolayer orientation (angle $\alpha$ $=0^{\circ}$ ) in the SEM images is determined by observing the patch shape and the monolayer orientation. The symmetric patch shape with a monolayer orientation as shown in Figure 1.B. is identified as the monolayer orientation with $\alpha=0^{\circ}$. The monolayer orientations of domains with different orientations shown in the other SEM images are determined with respect to the domain with the $\alpha=0^{\circ}$ monolayer orientation. The experimental values for $\theta$ and $\alpha$ are then used as parameters to obtain the patch shapes using the mathematical model.

Modeling: The patch geometry is obtained mathematically using a simple geometrical model where, the equations of the spheres (representing particles) and the inclined lines (representing incident rays) are solved simultaneously. The monolayer of the PS particles is described by hexagonally close-packed spheres sitting in the x-y plane. The vapors depositing on the sample are represented by the equations of lines hitting the $x-y$ plane at 
the angle of incidence $\theta$ and lying in the x-z plane. For a unit sphere diameter the density of the incident rays used for calculations is such that the rays are spaced by 0.0005 units in the $\mathrm{x}$ and $\mathrm{y}$ directions with respect to the $\mathrm{x}$-y plane. In our calculations we assume that the rays travel in a straight line parallel to each other and hit the sample at the angle of incidence, $\theta$. This assumption is based on the condition that the source is at an infinite distance with respect to the particle size, which is consistent with the experimental set up where the source distance to the sample $\left(\sim 10^{-1} \mathrm{~m}\right)$ can be treated as infinite compared to the particle size $\left(2.4 \cdot 10^{-6} \mathrm{~m}\right)$. For our calculations we consider a $6 \times 6$ sphere monolayer. The equation of each line is solved simultaneously with equations of the spheres with which it intersects. In the case where the ray intersects multiple spheres, the farthest $\mathrm{x}$ distance point (point closest to the source) is identified as the patchy point and the rest of the solutions are discarded. Solving the simultaneous equations of all the rays with the $6 \times 6$ sphere matrix gives solutions for the patchy geometries. To determine the exact patch boundary on the particle, the lines inclined at $\theta$ and tangent to the circles in the $\mathrm{x}-\mathrm{z}$ planes ( $\mathrm{x}-\mathrm{z}$ cross sectional view of the spheres are circles) are taken into account in addition to the grid of rays used for the patch area. MATLAB is used for solving the simultaneous equations and the code is available upon request. 